\title{
EDITORIAL
}

\section{Increased Helicobacter pylori-associated pathology in outbred mice coinfected with schistosomiasis}

\author{
Nashwa A. Elsaied • Aymn T. Abbas • \\ Samar N. El-Beshbishi • Hany M. Elsheikha
}

Received: 7 November 2008 / Accepted: 10 May 2009 / Published online: 26 May 2009

(C) Springer-Verlag 2009

To the Editor

Helicobacter pylori is a major gastroduodenal pathogen affecting about half of the world population (Taylor and Blaser 1991). It causes persistent gastritis and peptic ulceration and is directly linked to the development of various gastric malignant diseases (Huang et al. 1998; Uemura et al. 2001). Prevalence of $H$. pylori infection (Rowland et al. 2006) and the annual "true" reinfection rates (Zhang et al. 2009) are higher in developing countries than in developed countries and can vary by ethnicity, host genetics, place of birth, and socioeconomic factors. Differences in the incidence and severity of $H$. pylori infection can be attributed to differences in $H$. pylori strain virulence or the host's capacity to control H. pylori infection due to altered immune responses caused by concurrent infection and/or environmental factors (Kusters et al. 2006).

N. A. Elsaied

Department of Histology, Faculty of Veterinary Medicine,

Mansoura University,

Mansoura 35516, Egypt

\author{
A. T. Abbas \\ Gastroenterology Surgery Center, \\ Mansoura 35516, Egypt \\ S. N. El-Beshbishi \\ Department of Parasitology, Faculty of Medicine, \\ Mansoura University, \\ Mansoura 35516, Egypt \\ H. M. Elsheikha $(\square)$ \\ School of Veterinary Medicine and Science, \\ University of Nottingham, \\ Sutton Bonington Campus, \\ Leicestershire LE12 5RD, UK \\ e-mail: hany.elsheikha@nottingham.ac.uk
}

Helminth infections are estimated to infect three billion people worldwide (Crompton and Nesheim 2002). Schistosomiasis is helminth infection caused by the blood flukes of the genus Schistosoma. Despite intensive control efforts, disease caused by these worms remains a major public health concern in Egypt and +74 developing countries (Savioli et al. 1997). Although schistosomiasis is endemic in Egypt where H. pylori is a widespread problem and coinfections are frequent, limited data exist on the effects of schistosomiasis on the severity of $H$. pylori infection (Elshal et al. 2004). For the correct clinical management of $H$. pylori disease, obtaining thorough information on the other concurrent infections is essential. Here, we thought to compare the gastric pathologies in H. pylori-infected mice with concurrent Schistosoma mansoni infection with those infected with $H$. pylori alone.

Eight-week-old CD1 age-matched female outbred albino mice $(n=20)$ were obtained from Theodor Bilharz Research Institute, Cairo, Egypt. Mice were divided into four groups, each of five mice. Group (i) includes mice infected with $S$. mansoni and challenged with $H$. pylori 5 weeks after parasite infection, group (ii) includes mice challenged with $H$. pylori alone, group (iii) includes mice infected with $S$. mansoni alone, and group (iv) includes healthy noninfected control mice. Mice were infected with $60 \mathrm{~S}$. mansoni cercariae of an Egyptian strain as described (Elsheikha et al. 2008). For challenge with $H$. pylori, mice were inoculated intragastrically with a single dose of $0.1 \mathrm{ml}$ of Helicobacter suspension $\left(10^{7}\right.$ bacteria) and then observed for an additional 2 weeks. All mice were euthanized at 7 weeks post-S. mansoni infection (i.e., 2 weeks after bacterial infection). Animal infection was conducted according to protocols approved by the Animal Care and Ethics Committee at Mansoura University, Egypt. 
As shown in Fig. 1, all coinfected mice (group i) displayed a greater pathological alterations compared to those with a single $H$. pylori infection (group ii). The enhanced gastric pathological injury of coinfected mice was associated with a significant increase $(P$ value $<0.003)$ in parasite burden. Coinfected mice had more mean total number of recovered worms (19.8 \pm 1.6$)$ than did mice infected with $S$. mansoni alone $(14.6 \pm 1.1)$. Worms were not detected in the mesenteric veins of the infected mice. Apart from the presence of a few eggs in the intestinal mucosa associated with slight intestinal pathology, mice infected with $S$. mansoni did not show any other pathological changes. Guts of control mice showed a normal histological architecture without any pathological alterations. The increased pathology observed with concurrent $S$. mansoni and $H$. pylori infections demonstrate that the severity of $H$. pylori infection is exacerbated by the concurrent infection with $S$. mansoni and that schistosomiasis may be a risk factor for aggravated $H$. pylori pathogenicity. Although the circumstantial evidence is reasonable, it remains to be delineated whether this association translates into a causal relationship between concurrent schistosomiasis and gastric injuries in $H$. pylori infection.

The role of concurrent infections in ameliorating the disease severity has been known for many years. But, how may an existing helminth infection impair the host's ability to build up an adequate immune response to control concurrent or subsequent microbial infection and lead to aggravated pathology? This question has stimulated heated debate and contention over the past two decades. An imbalanced Th1/Th2 immune response (e.g., a crossregulatory suppression of Th1 response by a strong Th2 response) caused by helminth infection has been found to play a role in immune activation and/or dysregulation of the host immune response to concurrent bacterial infection (Brady et al. 1999; Mansfield et al. 2003). Although the Th1/Th2 imbalance theory after helminth infection has been suggested to play a role in immunopathology, in the real world when multiple infections coexist or vaccines are administered to coinfected individuals, the interactions between the responses induced between different types of organisms (helminths, bacteria, and viruses) become more sophisticated with unpredictable outcomes.

Evidences are conflicting on the effect of concurrent helminth infection on the immunopathogenesis and outcome of $H$. pylori infection. Some studies have shown that helminth infection may be protective against $H$. pylori infection and that infected patients may have a less severe form of the disease (Fox et al. 2000; Elshal et al. 2004). A popular example is the "African enigma" (high rates of infection and low gastric cancer rates) that has been explained in part by a disorder of the immune response caused by concurrent helminth infection (Holcombe 1992),
Fig. 1 Coinfection with the helminth parasite, S. mansoni, promotes $H$. pylori-mediated gastric pathological alterations in outbred mice. a At 2 weeks after infection with $H$. pylori only, the gastric mucosa and submucosa had mild neutrophilic infiltration (arrows). b-e At 7 weeks after $S$. mansoni infection and 2 weeks after coinfection with $H$. pylori (b) marked submucosal edema, congestion with inflammatory cells infiltration (arrow) at the basal layer of the fundic mucosa, (c) sever erosion up to ulceration of the surface mucosa with scattered inflammatory cells (arrow) within submucosa, (d) mild thinning and erosion of the surface crypts substituted with inflammatory cells (arrowheads), and (e) relative thinning and atrophy of the mucosa (arrow) with a small cluster of lymphoid follicles (arrowhead) in the submucosa (H\&E). Scale bars represent $200 \mu \mathrm{m}(\mathbf{a}, \mathbf{b})$ and $100 \mu \mathrm{m}(\mathbf{c}-\mathbf{e})$

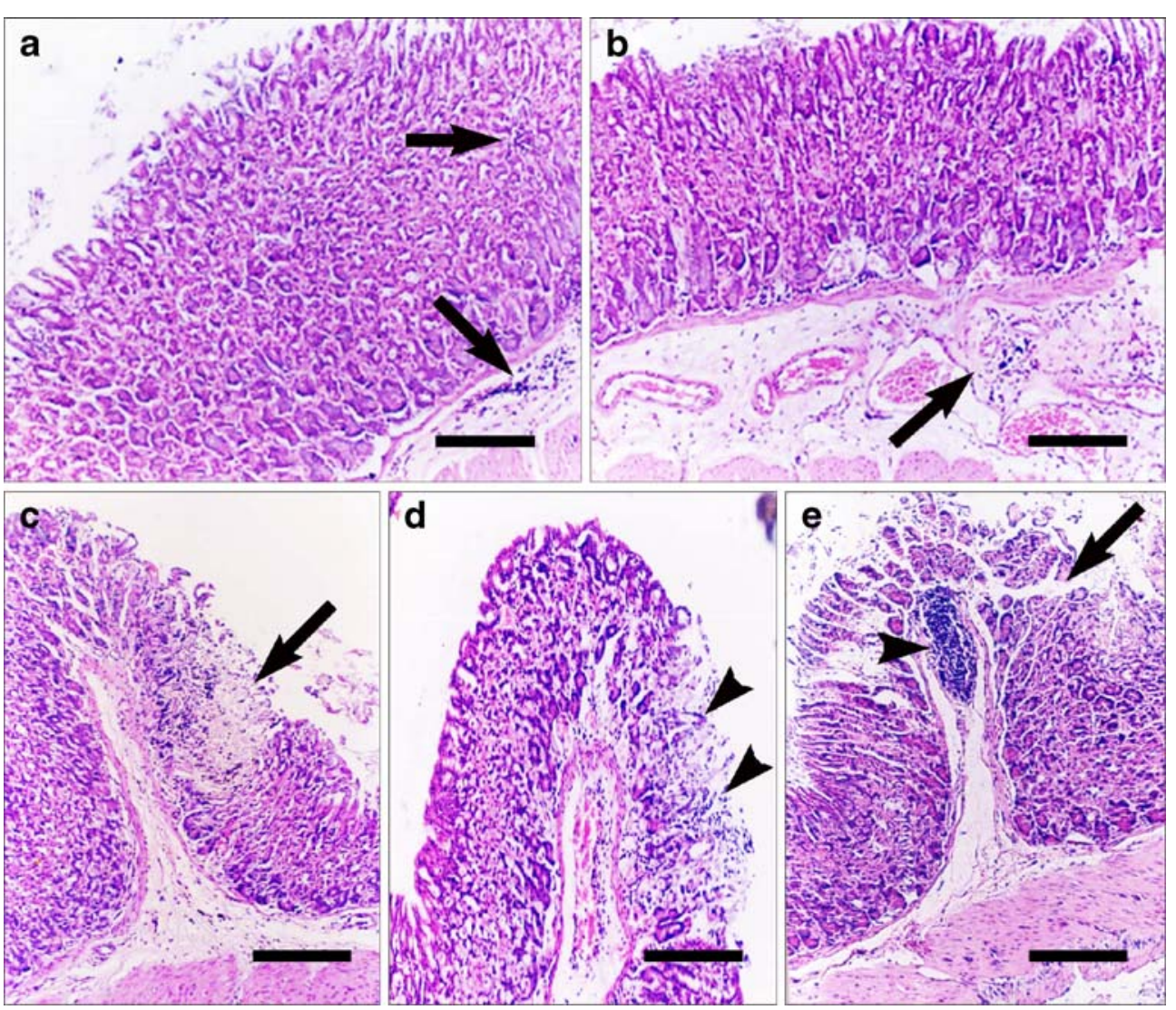


which effects a "switch" in Th1/Th2 polarity and confers a degree of protection. In contrast, Macdonald (2001) questioned the hypothesis of linking the African paradox phenomenon to the Th1/Th2 immune response imbalance caused by helminth infection and argued that the apparent protective effect of helminth infection is an incidental finding due to the experimental conditions of the study. Nonetheless, distinct patterns of $H$. pylori disease expression in different studies are more likely to be attributed to differential host inflammatory responses that influence pathologic outcome and/or may be related to the use of varied study designs.

Despite heterogeneity between studies, the prevalence of gut mucosal damage caused by $H$. pylori was significantly higher in mice with than without concurrent $S$. mansoni infection. These findings highlight the complexity of the immune circuitry coordinating the response of $H$. pyloril helminth coinfected individuals, the details of which are not fully known. Further well-designed immunopathogenesis studies and controlled trials are needed before any definitive conclusions regarding the clinical relevance of these findings in humans, particularly in therapy can be made.

\section{References}

Brady MT, O'Neill SM, Dalton JP, Mills KH (1999) Fasciola hepatica suppresses a protective Th1 response against Bordetella pertussis. Infect Immun 67:5372-5378

Crompton DWT, Nesheim MC (2002) Nutritional impact of intestinal helminthiasis during the human life cycle. Annu Rev Nutr 22:35-59

Elshal MF, Elsayed IH, El Kady IM, Badra G, El-Refaei A, ElBatanony M, Hendy OM (2004) Role of concurrent S. mansoni infection in $H$. pylori-associated gastritis: a flow cytometric
DNA-analysis and oxyradicals correlations. Clin Chim Acta 346:191-198

Elsheikha HM, Hussein HS, Rahbar MH (2008) Clinico-pathological effects of Schistosoma mansoni infection associated with simultaneous exposure to malathion in Swiss outbred albino mice. Acta Trop 108:11-19

Fox JG, Beck P, Dangler CA, Whary MT, Wang TC, Shi HN, NaglerAnderson C (2000) Concurrent enteric helminth infection modulates inflammation and gastric immune responses and reduces helicobacter-induced gastric atrophy. Nat Med 6:536542

Holcombe C (1992) Helicobacter pylori: the African enigma. Gut $33: 429-431$

Huang J-Q, Sridhar S, Chen Y, Hunt RJ (1998) Meta-analysis of the relationship between Helicobacter pylori seropositivity and gastric cancer. Gastroenterology 114:1169-1179

Kusters JG, van Vliet AH, Kuipers EJ (2006) Pathogenesis of Helicobacter pylori infection. Clin Microbiol Rev 19(3):449-490

Macdonald TT (2001) The worm turns on Helicobacter pylori. Gut 48:10-11

Mansfield LS, Gauthier DT, Abner SR, Jones KM, Wilder SR, Urban JF Jr (2003) Enhancement of disease and pathology by synergy of Trichuris suis and Campylobacter jejuni in the colon of immunologically naïve swine. Am J Trop Med Hyg 68:70-80

Savioli L, Renganathan E, Montresor A, Davis A, Behbehani K (1997) Control of schistosomiasis - a global picture. Parasitol Today 13:444-448

Taylor DN, Blaser MJ (1991) The epidemiology of Helicobacter pylori infection. Epidemiol Rev 13:42-59

Rowland M, Daly L, Vaughan M, Higgins A, Bourke B, Brumm B (2006) Age-specific incidence of Helicobacter pylori. Gastroenterology 130:65-72

Uemura N, Okamoto S, Yamamoto S, Matsumura N, Yamaguchi S, Yamakido M, Taniyama K, Sasaki N, Schlemper RJ (2001) Helicobacter pylori infection and the development of gastric cancer. N Engl J Med 345:784-789

Zhang YY, Xia HH, Zhuan ZH, Zhong J (2009) Review article: "True" re-infection of Helicobacter pylori after successful eradication: worldwide annual rates, risk factors and clinical implications. Aliment Pharmacol Ther 29:145-160 